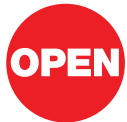

SUBJECT AREAS:

NONLINEAR OPTICS

OPTICAL MATERIALS AND STRUCTURES

ULTRAFAST PHOTONICS

FIBRE OPTICS AND OPTICAL COMMUNICATIONS

Received

5 July 2011

Accepted

1 September 2011

Published

15 September 2011

Correspondence and requests for materials should be addressed to R.K. (robert.keil@unijena.de)

\section{All-optical routing and switching for three-dimensional photonic circuitry}

\author{
Robert Keil', Matthias Heinrich', Felix Dreisow', Thomas Pertsch', Andreas Tünnermann', Stefan Nolte', \\ Demetrios N. Christodoulides² \& Alexander Szameit ${ }^{2}$
}

${ }^{1}$ Institute of Applied Physics, Friedrich-Schiller-Universität Jena, Max-Wien-Platz 1, 07743 Jena, Germany, ${ }^{2}$ College of Optics/ CREOL, University of Central Florida, 32816 Orlando, Florida, USA.

The ability to efficiently transmit and rapidly process huge amounts of data has become almost indispensable to our daily lives. It turned out that all-optical networks provide a very promising platform to deal with this task. Within such networks opto-optical switches, where light is directed by light, are a crucial building block for an effective operation. In this article, we present an experimental analysis of the routing and switching behaviour of light in two-dimensional evanescently coupled waveguide arrays of Y- and $\mathrm{T}$-junction geometries directly inscribed into fused silica using ultrashort laser pulses. These systems have the fundamental advantage of supporting three-dimensional network topologies, thereby breaking the limitations on complexity associated with planar structures while maintaining a high dirigibility of the light. Our results show how such arrays can be used to control the flow of optical signals within integrated photonic circuits.

nformation processing and networking schemes in all-optical circuits are expected to overcome the speed limitations associated with electronic system ${ }^{1}$ and can fully exploit the long-distance transmission capabilities of photons. Fundamental to such architectures is the rapid switching and routing of light signals in the optical domain. Due to steadily growing data volumes, one of the primary scopes of today's developments is to build integrated high-capacity devices with improved miniaturization. A widely acknowledged approach is the use of silicon as the optical material, since it offers a range of beneficial nonlinear properties ${ }^{2}$ and waveguides with minute radii of curvature become feasible due to its high refractive index $\mathrm{x}^{3,4}$. This allows for highly miniaturised devices, such as optical buffers ${ }^{5}$ and switches ${ }^{6,7}$, whereby the switching is actuated by optically induced refractive index changes and the switching speed is limited by free carrier generation. Other recent approaches employ silicon-organic hybrid waveguides for very fast signal processing ${ }^{8}$, slow light in coupled photonic crystal waveguides for all-optical switching on very short length scales $^{9}$ or use quantum dots to impose phase shifts determined by control fields at the single-photon level ${ }^{10}$.

Regardless of their physical platform, such components have to be cascaded for more complex operations. In a planar, i.e., non-intersecting, network, rerouting from $N$ inputs to $N$ outputs requires $N(N-1) / 2$ fundamental elements performing $2 \times 2$ switching, like a directional coupler ${ }^{11}$. Evidently, this $\mathbf{O}\left(N^{2}\right)$-scaling quickly becomes impractical as $N$ increases. It is by now quite clear that the existing two-dimensional (2D) planar technologies can only support a limited number of input/output channels since the demands on fabrication tolerances become progressively more severe with the number of nodes. This planar bottleneck can be broken by introducing the third (out-of-plane) dimension into the routing paradigm which allows a convenient low-loss cross-connection of the nodes leading to an $\mathrm{O}(N \log N)$-scaling ${ }^{12}$. In principle, a three-dimensional (3D) circuitry can be built from fundamental elements in which the light is routed within the plane perpendicular to the propagation axis of the waveguides. Ten years ago, such revolutionary elements were suggested in the form of two-dimensional (2D) junctions of evanescently coupled waveguide arrays ${ }^{13,14}$. To date, they could not be attempted experimentally in the optical domain due to a lack of appropriate materials and 3D waveguide fabrication technologies.

However, recent progress in the field of waveguide writing in glasses using ultrashort laser pulses ${ }^{15,16}$ has opened the gate for manufacturing arrays of waveguides with almost arbitrary arrangements in two transverse dimensions ${ }^{17,18}$. With this powerful and versatile technique we realised, for the first time, various all-optical routing and switching schemes in 2D waveguide arrays. We show that due to the interplay of linear and nonlinear light propagation, combined with polarisation as an additional degree of freedom, complex operations can be performed using simple settings. 


\section{Results}

Linear light propagation. In linear waveguide arrays ("photonic lattices"), light evanescently couples from one lattice site to the nearest neighbours, while propagating along the guides ${ }^{19}$. In particular, if the outermost waveguide is excited, the light forms a confined lobe that travels ballistically through the array ${ }^{20}$. In this work, such compact light distributions are used as signals. They can travel around sharp corners in the array, even perpendicularly to the initial transverse motion. As the light is propagating in the longitudinal direction, merely the small transverse momentum of light has to be changed to redirect the light signals. Hence, the light may pass corners without substantial losses, even for small refractive index contrasts. Such bends are described in the Supplementary Data \& Discussion. We focus here on the presentation of two fundamental building blocks of $2 \mathrm{D}$ routing and switching schemes: the Y- and T-junction (see Fig. 1a), where low-power signals couple into separate output ports and can be steered along defined paths by a high-power gating beam. In order to fabricate such structures, we employ the femtosecond (fs) laser direct-writing approach (see Methods). In Fig. 1c, three microscope images of the front facet of our samples (length $10 \mathrm{~cm}$, waveguide separation $32 \mu \mathrm{m}$ ) are shown, where the input positions of the low power signal and a high power gating beam are marked.

Discrete Solitons for Optical Routing and Switching. It is known that a high power beam, launched into a single site of a waveguide array, results in the formation of a nonlinearly trapped state, called "discrete soliton"19,21-25. Due to the optical Kerr effect, the effective refractive index of the respective guide is dynamically changed, resulting in a transient lattice defect, which follows the field intensity essentially instantaneously ${ }^{24}$. Evidently, such defects can be used to deflect optical signals and route them along defined paths $s^{13,14,26-28}$. In the first part of our experimental survey we investigated all-optical routing of linear signals by discrete solitons at $\mathrm{Y}$-junctions with an opening angle of $90^{\circ}$. The experimental results and the corresponding simulations are summarised in Fig. 2. In absence of the blocker, a weak signal $(\lambda=800 \mathrm{~nm}$, pulse duration $\sim 300 \mathrm{fs}$, pulse energy $\sim 50 \mathrm{~nJ}$, see Methods) couples equally into both arms of the Y-junction, as shown in the central column. We blocked one of the branches by launching high power laser pulses $(\lambda=800 \mathrm{~nm}$, pulse duration $\sim 300 \mathrm{fs}$, pulse energy $\sim 1 \mu \mathrm{J})$ into the waveguide adjacent to the junction. A pronounced spatial localisation of this gate pulse is visible in the first column, inducing the desired transient lattice defect, and thus effectively blocking that branch for the signal. The last column reveals how the path of the signal is altered by the presence of the blocker: The power transmitted into the blocked branch is reduced drastically and most of the signal light is steered into the remaining branch.

Beyond mere routing of signals, this scheme may also be used as a logic operator. To this end, we investigated optical switching on a waveguide $\mathrm{T}$-junction. The results, obtained with similar parameters as for the Y-junction, are summarised in Fig. 3. In absence of the blocker, most of the light is found in the vertical branch, a state which could be associated with a digital ' 1 ' in this port and a ' 0 ' in the horizontal branch. The blocker effectively diverts the signal beam into the horizontal branch, into a direction perpendicular to the initial direction of transverse motion, which would then yield the reverse output configuration. Due to the basically instantaneous nature of the Kerr nonlinearity the switching speed is only limited by the rate at which the blocker pulse itself is switched.
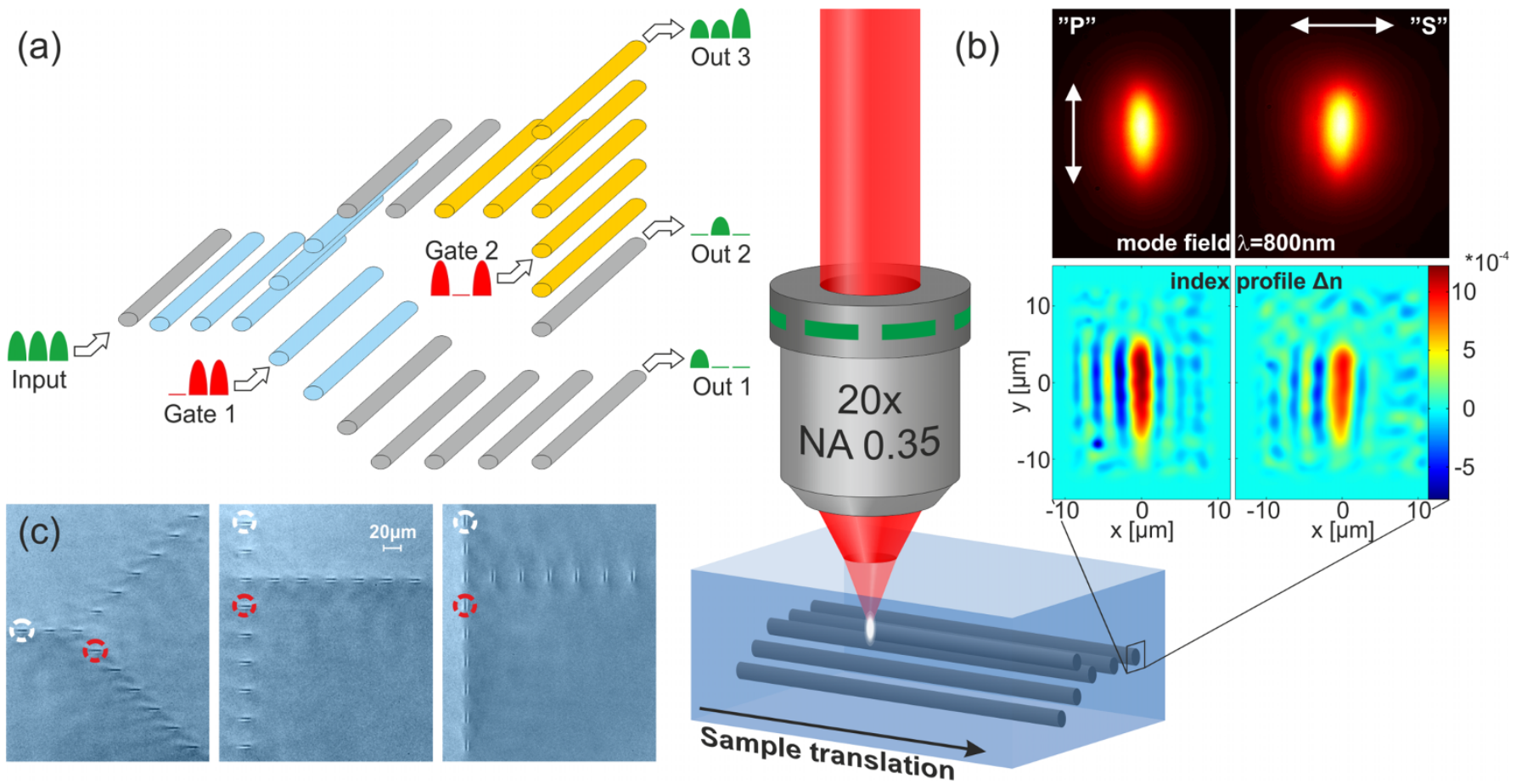

Figure $1 \mid$ Idea and setting of the proposed routing and switching scheme. (a) A 2D arrangement of evanescently coupled waveguides, which allows constraining light to travel along defined paths by opto-optical switching. The crucial Y-and T-junction segments are highlighted in blue and orange, respectively, and exemplary pulse sequences of the signal and the gating beam are shown in green and red. (b) Writing setup of waveguides using femtosecond laser pulses, which are focused into a bulk fused silica. The created refractive index change is dependent on the polarisation of the guided light, due to stress-induced birefringence of the material. Shown are resulting modal field distributions at $\lambda=800 \mathrm{~nm}$ and the corresponding refractive index profiles for s- and p-polarisation. (c) Micrographs of the front facet of two basic elements of this scheme, the Y-and the T-junction. Note that the T-junction is shown with two different orientations of the elliptical waveguides, harnessing polarisation as additional degree of freedom. The input positions of the high power blocker beam and the low power signal beam are marked with a red and a white circle, respectively. 
Blocker
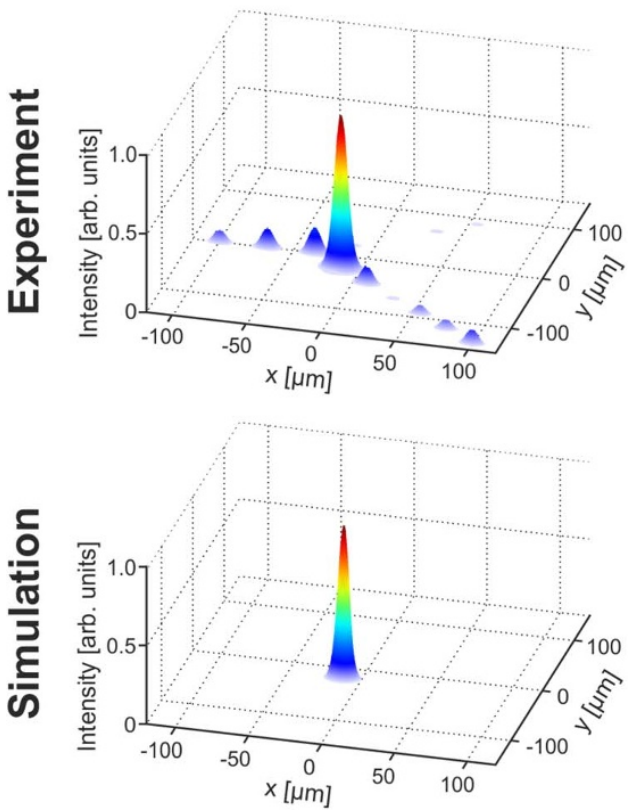

Signal w/o Blocker
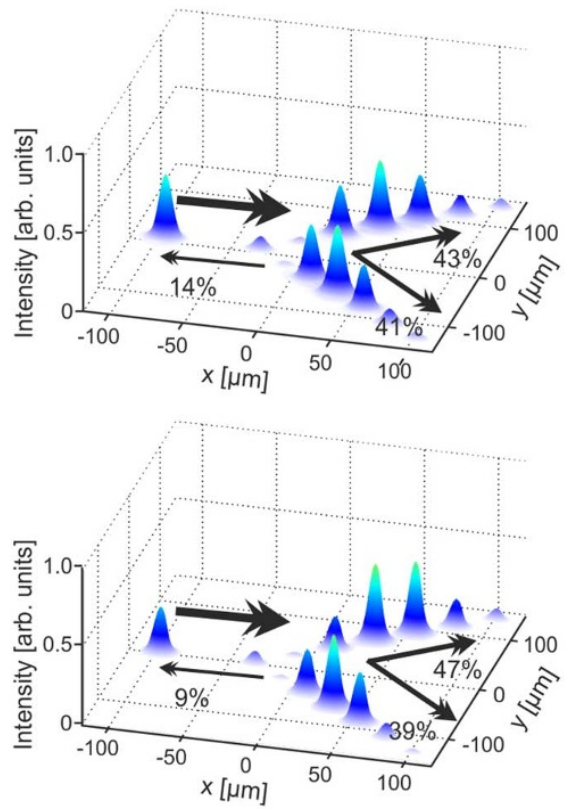

Signal w Blocker
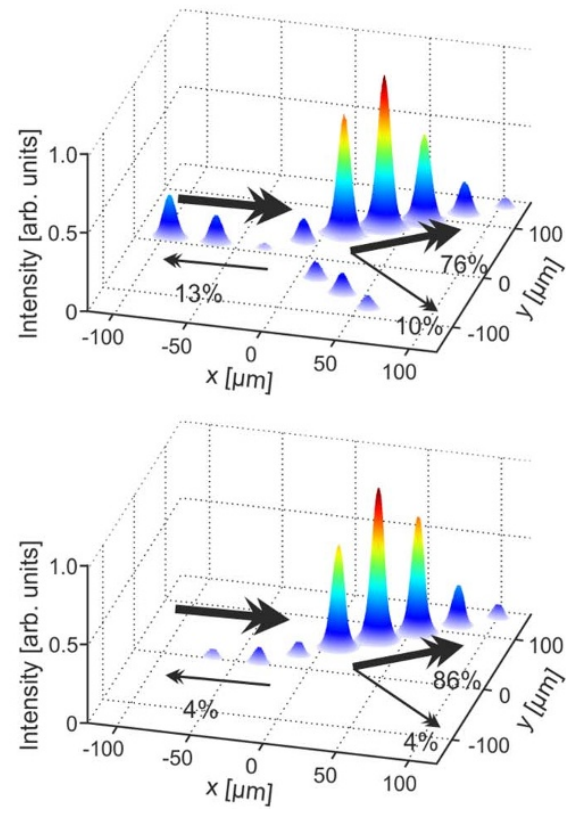

Figure $2 \mid$ All-optical routing at a Y-junction. Experiments (top row) and simulations (bottom row) at a Y-junction (left micrograph in Fig. 1c) showing the intensity distribution at the sample's output. Left column: The strongly localised high power beam, launched into the waveguide marked with a red circle in Fig. 1c. Central column: The propagation of a low power signal, launched into the waveguide marked with a white circle in Fig. 1c, when no blocker beam is present. Right column: Interaction of the two beams at the induced nonlinear defect; the lower branch gets blocked and the signal is routed into the upper port. Only the signal component is shown. In the experiment (simulation), a power ratio of $\sim 9 \mathrm{~dB}$ ( $14 \mathrm{~dB}$ ) was achieved between the two branches, compared to $0 \mathrm{~dB}(1 \mathrm{~dB})$ in absence of the gating pulse. Signal and Blocker intensities have been rescaled to their respective maximum. The thick arrow indicates the transverse motion of the input signal, while the smaller arrows and the percentage values show the relative power in each branch at the output of the device. For a full simulation of the light evolution see Movies S1-2 in the Supplementary Videos.

\section{Blocker}
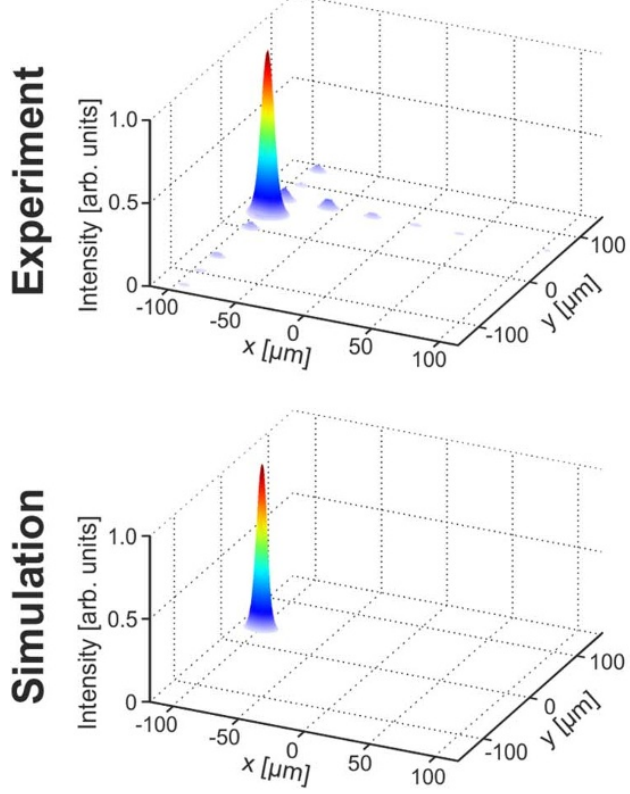

Signal w/o Blocker
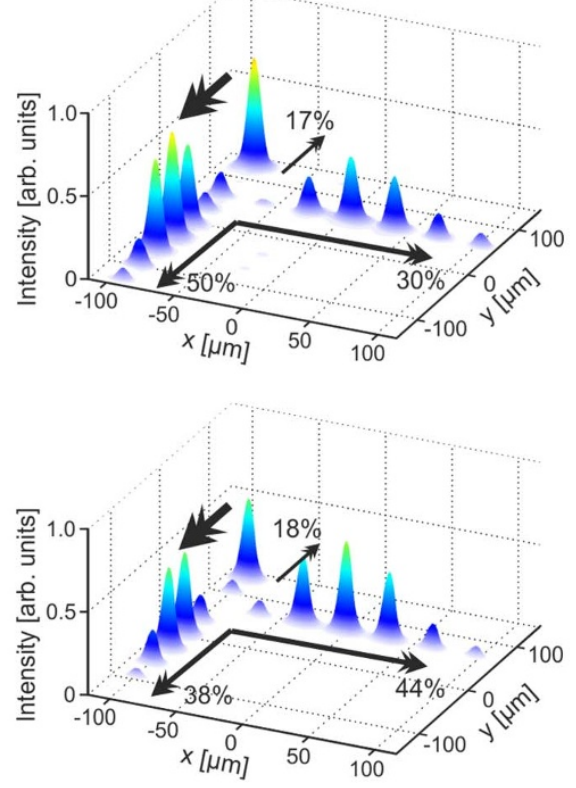

Signal w Blocker
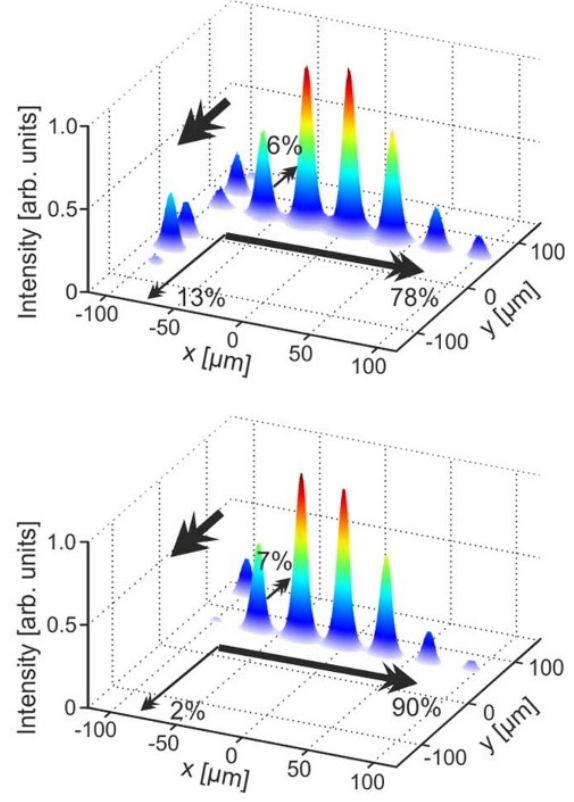

Figure 3 All-optical routing and switching at a T-junction. Experiments (top row) and simulations (bottom row) at a T-junction (central microscope image in Fig. 1c). Left column: Strongly localised high power beam, acting as blocker. Central column: The low power signal propagates into both branches with some preference for the forward direction. Right column: In presence of the blocker, the signal is routed into the transverse branch around an angle of $90^{\circ}$. The power ratio between the two branches is changed by $\sim 10 \mathrm{~dB}$ and only $\sim 6 \%$ of the light is reflected. In the cw-simulation the power ratio is changed by $\sim 15 \mathrm{~dB}$. Signal and Blocker intensities have been rescaled to their respective maximum. For a full simulation of the light evolution see Movies S3-4 in the Supplementary Videos. 
Polarisation selective routing. The presented optical switching concept can be extended by polarisation selective routing. To demonstrate this feature of our proposed scheme, we rotated the ellipses of the waveguides by $90^{\circ}$, as shown in the right microscope image of Fig. 1c, and measured the output intensity distributions for both input polarisations (termed s- and p-polarisation). Due to the modal shape of the beams at different polarisations (Fig. 1b), the output patterns become strongly polarisation dependent, in contrast to our results presented in Figs. 2 and 3. In absence of the blocker the s-polarised signal couples into both output branches (Fig. 4a). In case of p-polarisation the light moves straight across the junction as the coupling in the perpendicular direction is weaker than in the forward one (Fig. 4c). If the first waveguide of the forward path is blocked, s-polarised light is mainly routed into the side branch (Fig. 4b), whereas a p-polarised signal is reflected back into the input port, due to the p-specific coupling conditions (Fig. 4d). Thereby, the device acts as an all-optically switched polarisation filter, separating polarisations on demand.

\section{Discussion}

Our results demonstrate how discrete solitons can be used to effectively route and switch light signals at junctions of evanescently coupled waveguide arrays. Non-planar network topologies are supported by such routing and switching elements.

Note that the waveguide arrays investigated in this survey are merely weakly coupled due to their relatively large separation $(32 \mu \mathrm{m})$, which has been chosen for experimental convenience. To allow for circuits combining several elements on the same length, as depicted in Fig. 1a, one needs to reduce the waveguide separation, and thereby increase the coupling, which is well possible by one order of magnitude for directly written waveguides in fused silica ${ }^{29}$.
We would like to emphasise the close correspondence of the numerical results and the experimental data shown in Figs. 2 and 3. The only major difference between the two is the stronger localisation of the blocker beam in the simulations, which were carried out assuming continuous wave (cw) illumination (see Methods). On the other hand, the experiments are subjected to the spatio-temporal dynamics inherent to a pulsed excitation, whereby the low-power slopes of the pulse do not contribute to localisation ${ }^{30}$. Therefore, the lower switching contrast in the experiment can be attributed to the temporal dynamics of the gate pulse propagation. To improve the performance of the junctions beyond these proof-of-principle values ( $\sim 10 \mathrm{~dB}$ for the $\mathrm{T}$-junction), one can use longer gate pulses, and thereby an increased interaction window. Then, switching contrasts of $\sim 15 \mathrm{~dB}$, as obtained from the simulations, can be expected. A further enhancement is possible by shifting the blocker pulse between the first waveguides of the two output branches instead of merely chopping its amplitude.

It is also insightful to have a look at the reflection loss for the signal at the junctions. Comparing to the data found in simple bends (see Supplementary Data \& Discussion, Fig. S1), one finds that considerably more light is reflected at the junctions (Figs. 2 and 3, central column). This arises from an additional topological defect caused by the branching of the array ${ }^{31}$. However, this defect is overcome when the blocker beam is present, as the lower branch effectively vanishes for the signal. Hence, the remaining branches are equivalent to a bent array and exhibit a similarly weak reflection.

However, when it comes to building larger networks out of cascades of such elementary junctions, even small reflection losses are crucial. For instance, at the gated T-junction about $6 \%$ of the power radiates back into the input port, whereas $17 \%$ is lost in the ungated case. Such reflection losses can be mitigated by a detuning of the

\section{Signal w/o Blocker}

(a)

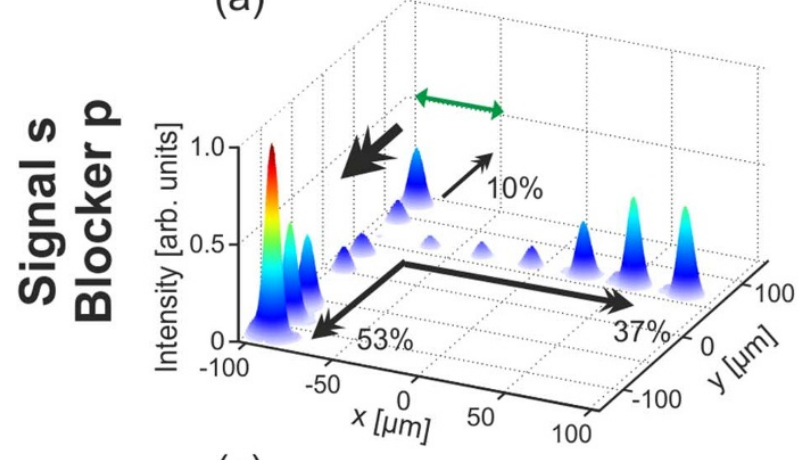

(c)

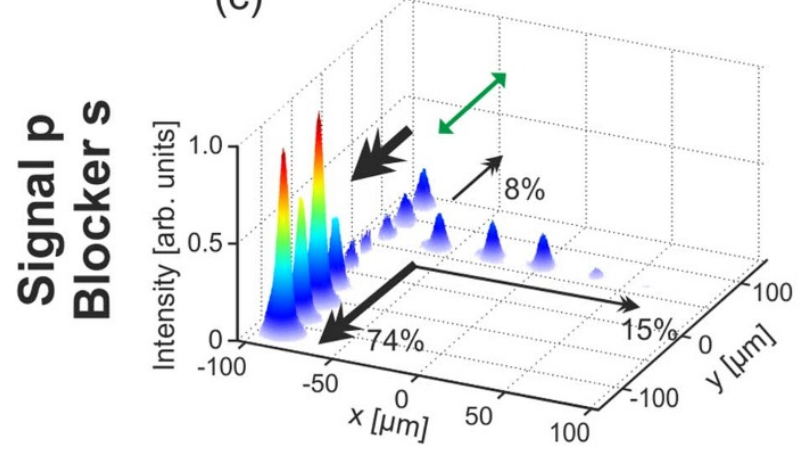

(b)

\section{Signal w Blocker}

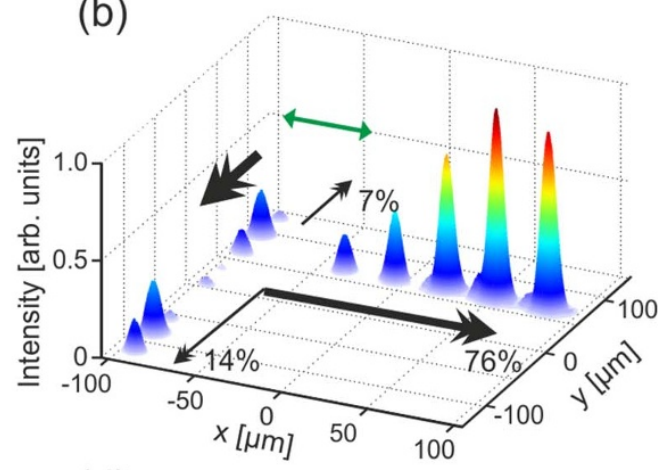

(d)

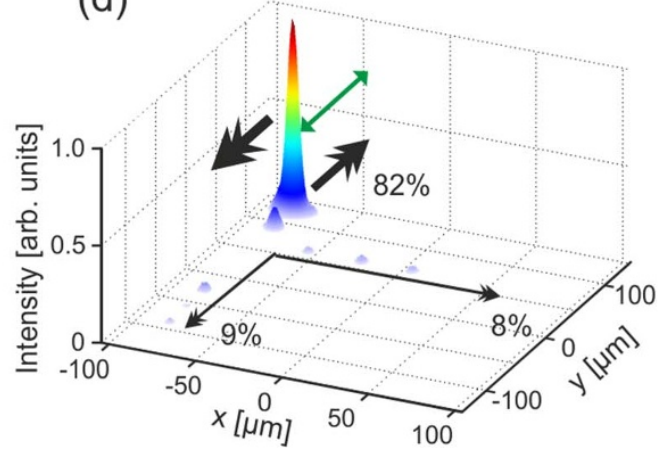

Figure 4 | Measured polarisation-dependent all-optical routing and switching at a T-junction. (a) An s-polarised low-power signal couples into both output branches when no blocker is present. (b) Activating the high power blocker beam results in deflection of the signal into the perpendicular branch of the T-junction (power ratio altered by $\sim 9 \mathrm{~dB}$ ). (c) A p-polarised low-power signal travels straight when no blocker is present. (d) The blocker beam causes back-reflection from the pivotal point into the input port, such that almost no light is deflected into the perpendicular branch (power ratio changed by $\sim 13 \mathrm{~dB}$ ). Note that each image has been rescaled to its maximum. The green arrow indicates the signal polarisation. 
pivotal waveguide, as it has been numerically $y^{32}$ and experimentally ${ }^{33}$ shown for bent arrays.

The propagation loss in the laser-written waveguides is in the order of $0.4 \mathrm{~dB} / \mathrm{cm}$, amounting to a global loss of $60 \%$ over the sample length. However, for a networking device one would use stronger coupling $^{29}$, meaning that more junctions can be accommodated on a given propagation length. Also note, that losses as low as $0.05 \mathrm{~dB} / \mathrm{cm}$ have been reported for fs-laser written waveguides after thermal treatment ${ }^{34}$.

It may be of interest to compare the presented laser-written waveguide array junctions with similar structures constituted by photonic crystal waveguides. For example, integrated MachZehnder interferometers have been realised, with a thermal ${ }^{35}$ or all-optical ${ }^{36}$ control of the light propagation. Such structures can be much more miniaturised; due to their enormous refractive index contrast, the light is normally confined to the $\mu \mathrm{m}$-range, whereas the transverse dimension of our splitter is $\sim 100 \mu \mathrm{m}$. Interestingly, the reflection loss at a Y-splitter is predicted to be similar to the loss occurring at our devices, if the splitters are engineered accordingly ${ }^{37}$. Even though propagation losses are much higher for photonic crystal waveguides $\left(\sim 8 \mathrm{~dB} / \mathrm{cm}^{38}\right)$, the much smaller propagation lengths (some $10 \ldots 100 \mu \mathrm{m}$ per device instead of $1 \ldots 10 \mathrm{~cm}$ ) more than compensate for it.

On the other hand, these remarkable properties come usually at the expense of a narrow bandwidth, whereas devices relying on directional coupling in laser written waveguides have been demonstrated to operate for spectra spanning more than an octave ${ }^{39}$. Moreover, all photonic crystal waveguides reported so far were entirely planar. Networks constructed out of them will thereby suffer from the aforementioned disadvantageous scaling properties $^{11}$. Finally, the nonlinear response time is not limited in fused silica $^{24}$, at the cost of requiring relatively large energies for the gate pulse.

Therefore, photonic crystal devices will be superior when it is desired to realise highly miniaturised, low-loss, energy-efficient networks with a moderate number of elements for signals at a predetermined spectral range and where speed is not absolutely critical. For broadband applications as well as for very large networks, where the better scaling of $3 \mathrm{D}$ topologies becomes relevant ${ }^{12}$, or when the speed of operation is more important than energy-efficiency, the waveguide array devices presented in this manuscript may provide a very useful alternative.

Notably, such routing and switching schemes in waveguide arrays may allow for a variety of further all-optical devices for communication and information processing. In Fig. 5 two different examples are given. The left column shows an optically gatable wavelength splitter. The coupling strength between adjacent guides, and hence, the transverse velocity of signals, increases with wavelength ${ }^{29}$. We envision a Y-junction where the high-power pulse can swap sides via curved waveguide sections. In such a $3 \mathrm{D}$-device the signal may be split into its spectral components depending on the input position of the blocker pulse. A further possibility is the realisation of a (classical) all-optical controlled-NOT (CNOT) gate (right column of Fig. 5). The high-power pulse controls the gate, by blocking the signal and preserving the target bit if sent into the centre of the structure (Fig. $5 \mathrm{c}$ ) or flipping the bit if propagating in the decoupled waveguide (Fig. 5d). Alternatively, a nonlinear CNOT gate could be constructed where the target bit is set by the control beam's power.

In conclusion, we experimentally studied $2 \mathrm{D}$ all-optical routing and switching schemes in fs laser-written waveguide arrays. The investigated structures can be combined to complex non-planar photonic networks in which discrete solitons direct light signals along specific paths. The presented approach suggests a new concept of building purely optical devices which may be used for a rapid routing and processing of data in form of optical pulses. The
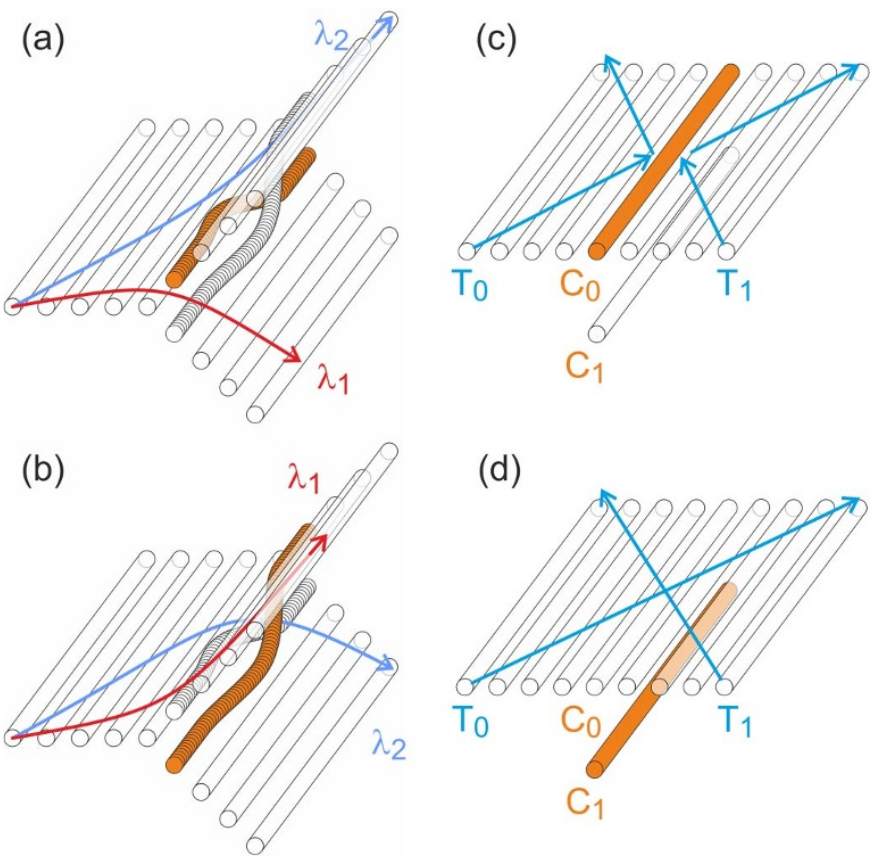

(d)

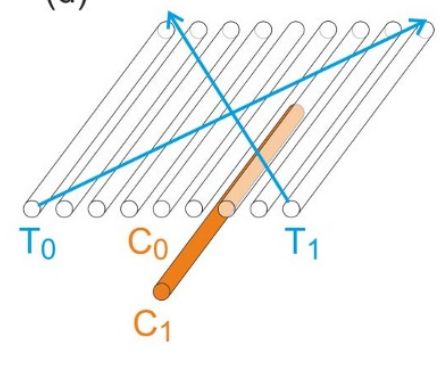

Figure $5 \mid$ Proposition of other integrated all-optical devices.

(Left column) Wavelength splitter based on the wavelength dependence of the coupling strength. (a) The blocker pulse (orange) is shifted from the upper branch to the lower branch at the curved waveguide segments. Consequently, the signal with longer wavelength $\left(\lambda_{1}\right)$ is routed into the lower branch and the signal of shorter wavelength $\left(\lambda_{2}<\lambda_{1}\right)$ is routed into the upper branch since the blocker occurs on the other side in the second section of the structure. (b) The blocker pulse is launched into the opposite waveguide to reverse the situation. (Right Column) All-Optical CNOT gate. (c) The controlling blocker deflects the target signals, forcing them to remain in their respective channel. (d) The target-bits are swapped if the blocker is sent into the decoupled channel.

instantaneous nature of the Kerr nonlinearity sets no fundamental limits to the achievable speed of operation.

\section{Methods}

Direct waveguide writing. We used the femtosecond (fs) laser direct-writing technique to fabricate our devices. Femtosecond laser pulses from a Ti:Sapphire laser system (Coherent Mira/RegA; wavelength $\lambda=800 \mathrm{~nm}$, pulse length $170 \mathrm{fs}$, pulse energy $\sim 300 \mathrm{~nJ}$, repetition rate $100 \mathrm{kHz}$ ) are focused into a transparent bulk material. Since the required energy densities for the underlying nonlinear absorption process are only reached in the focal spot of the writing objective $(\mathrm{NA}=0.35)$, the resulting modifications are confined to a small volume. In case of fused silica as host material, illumination with fs pulses yields a localised refractive index increase in the order of $10^{-3}$. Consequently, an elongated waveguide following an arbitrary trajectory can be created by moving the sample with respect to the beam (velocity $1.5 \mathrm{~mm} / \mathrm{s}$ ). Since the guides are written sequentially, extended arrangements with intricate geometries can be realised (see Fig. 1b). In addition, our proposed scheme makes use of the stress-induced birefringence of the waveguides, which causes light with different polarisation (termed s- and p-polarisation) to exhibit slightly different mode shapes.

Experimental routing \& switching setup. To perform all-optical routing and switching in the experiment, two pulses from a Ti:Sapphire laser system (Spectra Physics Spitfire; $\lambda=800 \mathrm{~nm}$, pulse duration $\sim 300 \mathrm{fs}$ ) were simultaneously launched through a microscope objective $(\mathrm{NA}=0.075)$ into the array-junctions. A MachZehnder interferometer with adjustable path-length and combinations of half-wave plates and polarisers was used to overlap the pulses temporally and to select input sites, pulse energies and polarisations for both pulses individually (For more details see Supplementary Methods and Supplementary Fig. S2). The output intensity profile at the end facet of the sample was imaged onto a CCD. The beams were orthogonally polarised, such that the contributions of the two pulses could be measured separately by positioning an additional polariser behind the sample.

Simulating the field propagation. The paraxial Helmholtz equation including a Kerr nonlinearity governing the propagation of two orthogonally polarised modal field 
amplitudes $A(x, y, z)$ and $B(x, y, z)$, assuming material birefringence, $|B| \gg|A|$ and slow variation in $z$-direction, is given by:

$$
\begin{aligned}
& i \frac{\partial A(x, y, z)}{\partial z}=-\left[\frac{1}{2 \beta_{A}} \nabla_{x, y}^{2}+\frac{k_{0}^{2} n_{A}^{2}(x, y)-\beta_{A}^{2}}{2 \beta_{A}}+\gamma_{X P M}|B|^{2}\right] A(x, y, z) \\
& i \frac{\partial B(x, y, z)}{\partial z}=-\left[\frac{1}{2 \beta_{B}} \nabla_{x, y}^{2}+\frac{k_{0}^{2} n_{B}^{2}(x, y)-\beta_{B}^{2}}{2 \beta_{B}}+\gamma_{S P M}|B|^{2}\right] B(x, y, z)
\end{aligned}
$$

where $k_{0}=\frac{2 \pi}{2}$ is the vacuum wavenumber, $\beta_{A(B)}$ denotes the propagation constant of the respective waveguide mode and $n_{A(B)}(x, y)$ the transverse refractive index profiles. The terms on the right hand side account for diffraction, the contribution of the trapping index profile and the Kerr nonlinearity, respectively. Thereby, the parameters of self- and cross-phase modulation are given by $\gamma_{S P M}=\frac{n_{0} \varepsilon_{0} c}{2} k_{0} n_{2}$ and $\gamma_{X P M}=\frac{2}{3} \gamma_{S P M}$ for fused silica, with an intensity dependent refractive index $n(I)=n_{0}+n_{2} I\left(n_{0}=1.45, n_{2}=3.2 \times 10^{-20} \mathrm{~m}^{2} \mathrm{~W}^{-1}\right)$ We employed a Fourier-splitstep algorithm ${ }^{40}$ to simulate the propagation of light through various waveguide arrays. The mode profiles of the fs laser-written waveguides were measured for both polarisations to obtain the corresponding index profiles by inverting the scalar linear Helmholtz equation ${ }^{41}$, neglecting form birefringence of the waveguides. Note that this approximation does not affect the simulation of the light propagation, as the correct modal profiles are recovered from these two index distributions again. The waveguides are found to have a maximum index of $1.2 \times 10^{-3}$ above the bulk material for a p-polarised field and $9.8 \times 10^{-4}$ for s-polarisation (Fig. 1b). Several of these profiles were concatenated to model Y-and T-junctions of waveguide arrays. Movies of the switching operations are presented in Supplementary Videos 1-4.

1. Kivshar, Y. S. Nonlinear optics: The next decade. Opt. Express 16, 22126-22128 (2008).

2. Leuthold, J., Koos, C. \& Freude, W. Nonlinear silicon photonics. Nature Phot. 4, 535-544 (2010).

3. Vlasov, Y. \& McNab, S. J. Losses in single-mode silicon-on-insulator strip waveguides and bends. Opt. Express 12, 1622-1631 (2004).

4. Cardenas, J. et al. Low loss etchless silicon photonic waveguides. Opt. Express 17, 4752-4757 (2009).

5. Xia, F., Sekaric, L. \& Vlasov, Y. Ultracompact optical buffers on a silicon chip. Nature Phot. 1, 65-71 (2007).

6. Almeida, V. R., Barrios, C. A., Panepucci, R. R. \& Lipson, M. All-optical control of light on a silicon chip. Nature 431, 1081-1084 (2004).

7. Vlasov, Y., Green, W. M. J. \& Xia, F. High-throughput silicon nanophotonic wavelength-insensitive switch for on-chip optical networks. Nature Phot. 2, 242246 (2008).

8. Koos, C. et al. All-optical high-speed signal processing with silicon-organic hybrid slot waveguides. Nature Phot. 3, 216-219 (2009).

9. Kampfrath, T. et al. Ultrafast rerouting of light via slow modes in a nanophotonic directional coupler. Appl. Phys. Lett. 94, 241119 (2009).

10. Fushman, I. et al. Controlled phase shifts with a single quantum dot. Science $\mathbf{3 2 0}$, 769-772 (2008).

11. Spanke, R. A. \& Benes, V. E. N-stage planar optical permutation network. Appl. Optics 26, 1226-1229 (1987).

12. Benes, V. E. Growth, complexity, and performance of telephone connecting networks. Bell Syst. Tech. J. 62, 499-539 (1983).

13. Christodoulides, D. N. \& Eugenieva, E. D. Blocking and routing discrete solitons in two-dimensional networks of nonlinear waveguide arrays. Phys. Rev. Lett. 87, 233901 (2001).

14. Eugenieva, E. D., Efremidis, N. K. \& Christodoulides, D. N. Design of switching junctions for two-dimensional discrete soliton networks. Opt. Lett. 26, 1978-1980 (2001).

15. Itoh, K., Watanabe, W., Nolte, S. \& Schaffer, C. Ultrafast processes for bulk modification of transparent materials. MRS Bulletin 31, 620-625 (2006).

16. Nolte, S., Will, M., Burghoff, J. \& Tünnermann, A. Femtosecond waveguide writing: a new avenue to three-dimensional integrated optics. Appl. Phys. A 77, 109-111 (2003).

17. Szameit, A. et al. Hexagonal waveguide arrays written with fs-laser pulses. Appl. Phys. B 82, 507-512 (2006).

18. Pertsch, T. et al. Discrete diffraction in two-dimensional arrays of coupled waveguides in silica. Opt. Lett. 29, 468-470 (2004).

19. Christodoulides, D. N., Lederer, F. \& Silberberg, Y. Discretizing light behaviour in linear and nonlinear waveguide lattices. Nature 424, 817-823 (2003).
20. Makris, K. G., Suntsov, S., Christodoulides, D. N., Stegeman, G. I. \& Hache, A. Discrete surface solitons. Opt. Lett. 30, 2466-2468 (2005).

21. Christodoulides, D. N. \& Joseph, R. I. Discrete self-focusing in nonlinear arrays of coupled waveguides. Opt. Lett. 13, 794-796 (1988).

22. Eisenberg, H. S., Silberberg, Y., Morandotti, R., Boyd, A. R. \& Aitchison, J. S. Discrete spatial optical solitons in waveguide arrays. Phys. Rev. Lett. 81, 33833386 (1998).

23. Fleischer, J. W., Segev, M., Efremidis, N. K. \& Christodoulides, D. N. Observation of two-dimensional discrete solitons in optically induced nonlinear photonic lattices. Nature 422, 147-150 (2003).

24. Lederer, F. et al. Discrete solitons in optics. Phys. Rep. 463, 1-126 (2008).

25. Szameit, A. et al. Discrete nonlinear localization in femtosecond laser written waveguides in fused silica. Opt. Express 13, 10552-10557 (2005).

26. Bang, O. \& Miller, P. D. Exploiting discreteness for switching in waveguide arrays. Opt. Lett. 21, 1105-1107 (1996).

27. Meier, J. et al. Beam interactions with a blocker solitons in one-dimensional arrays. Opt. Lett. 30, 1027-1029 (2005).

28. Meier, J. et al. Incoherent blocker soliton interactions in Kerr waveguide arrays. Opt. Lett. 30, 3174-3176 (2005).

29. Szameit, A., Dreisow, F., Pertsch, T., Nolte, S. \& Tünnermann, A. Control of directional evanescent coupling in fs laser written waveguides. Opt. Express 15, 1579-1587 (2007).

30. Heinrich, M. et al. Observation of three-dimensional discrete-continuous X waves in photonic lattices. Phys. Rev. Lett. 103, 113903 (2009).

31. Heinrich, M. et al. Nonlinear localized states in the vicinity of topological defects in waveguide arrays. New J. Phys. 12, 113020 (2010).

32. Christodoulides, D. N. \& Eugenieva, E. D. Minimizing bending losses in twodimensional discrete soliton networks. Opt. Lett. 26, 1876-1878 (2001).

33. Heinrich, M. et al. Nonlinear discrete optics in femtosecond laser-written photonic lattices. Appl. Phys. B 104, 469-480 (2011).

34. Fukuda, T., Ishikawa, S., Fujii, T., Sakuma, K. \& Hosoya, H. Low-loss optical waveguides written by femtosecond laser pulses for three-dimensional photonic devices. Proc. SPIE 5339, 524-538 (2004).

35. Vlasov, Y. A., O’Boyle, M., Hamann, H. F. \& McNab, S. J. Active control of slow light on a chip with photonic crystal waveguides. Nature 438, 65-69 (2005).

36. Asakawa, K. et al. Photonic crystal and quantum dot technologies for all-optical switch and logic device. New J. Phys. 8, 208 (2006).

37. Krauss, T. F. Planar photonic crystal waveguide devices for integrated optics. Phys. Stat. Sol. A 197, 688-702 (2003).

38. Dulkeith, E., McNab, S. J. \& Vlasov, Y. A. Mapping the optical properties of slabtype two-dimensional photonic crystal waveguides. Phys. Rev. B 72, 115102 (2005).

39. Dreisow, F. et al. Polychromatic beam splitting by fractional stimulated Raman adiabatic passage. Appl. Phys. Lett. 95, 261102 (2009).

40. Agrawal, G. P. Nonlinear Fiber Optics (Academic Press, San Diego, ed. 3 2001).

41. Mansour, I. \& Caccavale, F. An improved procedure to calculate the refractive index profile from the measured near-field intensity. J. Lightwave Technol. 14, 423-428 (1996).

\section{Acknowledgements}

The authors acknowledge support by the Deutsche Forschungsgemeinschaft (Research Unit 532 and Leibniz program) and the German Academy of Science Leopoldina (grant LPDS 2009-13).

\section{Author Contributions}

R.K., M.H., F.D., T.P., S.N. and A.S. devised the experimental setup and performed the measurements. R.K., M.H. and A.S. analysed the results and prepared the figures. F.D. fabricated the samples. R.K., F.D., D.C. and A.S. engaged in modelling and numerical simulations. All authors co-wrote the paper.

\section{Additional information}

Supplementary information accompanies this paper at http://www.nature.com/ scientificreports

Competing financial interests: The authors declare no competing financial interests.

License: This work is licensed under a Creative Commons

Attribution-NonCommercial-NoDerivative Works 3.0 Unported License. To view a copy of this license, visit http://creativecommons.org/licenses/by-nc-nd/3.0/

How to cite this article: Keil, R. et al. All-optical routing and switching for three-dimensional photonic circuitry. Sci. Rep. 1, 94; DOI:10.1038/srep00094 (2011). 